EPJ Web of Conferences 41, 07022 (2013)

DOI: $10.1051 /$ epjconf/20134107022

(C) Owned by the authors, published by EDP Sciences, 2013

\title{
Time-Resolved Down-Conversion of 2-Aminopurine in a DNA Hairpin: Fluorescence Anisotropy and Solvent Effects
}

\author{
Patricia Tourón Touceda, Thomas Gelot, Olivier Crégut, Jérémie Léonard and Stefan Haacke \\ Institut de Physique et Chimie des Matériaux de Strasbourg, Université de Strasbourg-CNRS, 67034 \\ Strasbourg, France
}

\begin{abstract}
Femtosecond fluorescence anisotropy decay measured by type II difference frequency generation provides new insight into the local structural dynamics of $\triangle \mathrm{P}(-) \mathrm{PBS}$ fragments of the HIV- 1 DNA primary binding sequence, labeled with 2aminopurine.
\end{abstract}

\section{Introduction}

Structural dynamics and heterogeneity of biomolecules are intimately related to their functions. The flexibility of DNA was shown to play an important role for many processes, such as protein binding, transcription or packaging. Characterization of such localized structural changes typically requires the use of spectroscopic methods in which environment-sensitive fluorescent analogues of natural nucleobases are incorporated into DNA at specific positions. As such, 2-Aminopurine (2-AP) has been shown to minimally perturb DNA structure and stability. 2-AP can be selectively excited at wavelengths between 300 and $330 \mathrm{~nm}$, where natural bases do not absorb. The peak of 2-AP fluorescence in aqueous solutions is at $371 \mathrm{~nm}$, with a single decay constant of $10.4 \mathrm{~ns}$. When incorporated to DNA, 2-AP fluorescence is extensively quenched [1-3].

We investigate the structural heterogeneity and dynamics of $\triangle \mathrm{P}(-) \mathrm{PBS}$, a fragment of the HIV Primary Binding Site DNA hairpin. The oligonucleotide is labelled with 2-AP in positions 6, 8 or 10 (see fig. 1a), and we measure the ultrafast, UV fluorescence decay by implementing type II, difference frequency generation (DFG) in BBO with 40-fs 800-nm gate pulses [4]. Recently we have confirmed that the strong quenching of 2-AP fluorescence is highly site-specific [5]. For each oligonucleotide, four decay times were identified, differing by almost an order of magnitude: 1-3ps, 10-40ps, 100-150 ps and $>2 \mathrm{~ns}$, with site-specific amplitudes [6]. We attributed the lifetimes to qualitatively distinct classes of local conformations of 2-AP with respect to its nearest neighbor nucleobases. The shortest sub-5- ps decay times were assigned to ground-state stacking interactions of 2-AP with guanine $(\mathrm{G})$. While the longest nanosecond component is attributed to collisional quenching with $\mathrm{G}$, the two intermediate quenching times deserved further investigation.

To this end, we have measured time-resolved fluorescence anisotropy, and the effect of solvent viscosity on the overall quenching dynamics. The orientational dynamics of the 2-AP transition dipole moment is governed by tumbling of the entire oligonucleotide on a 2-3 ns time scale, but also by faster segmental base motions [5].

This is an Open Access article distributed under the terms of the Creative Commons Attribution License 2.0, which permits unrestricted use, distribution, and reproduction in any medium, provided the original work is properly cited. 


\section{Materials and Methods}

Solutions of 2-AP-labeled $\triangle \mathrm{P}(-) \mathrm{PBS}$ oligonucleotides and free 2-AP were prepared as described in [4]. The down-conversion setup has been described in $[4,6]$. The polarization of the excitation beam was linear and set to magic angle with respect to the BBO crystal axis to measure the excited state population decay. It was set to parallel or perpendicular to record successively the two DFG signals used to compute the anisotropy decay. Small drifts and fluctuations in the excitation power were recorded in parallel and corrected for.

\section{Results and Discussion}

Figure 1d shows typical fluorescence anisotropy decay curves for the three hairpins, recorded at the fluorescence central wavelength. As expected, the anisotropy decay is much slower than that of free 2-AP (see fig. 1b and d), and does not decay to zero within the available time window. In addition, unlike the mono-exponential anisotropy decay of free 2-AP, the oligonucleotides data exhibit a bi-exponential decay (see fig. 1b), giving evidence for structural dynamics occurring on at least two different length scales, characterizing the local structural flexibility. Remarkably, the anisotropy traces for the three hairpins are very similar and present a fast component, in the order of $\approx 100 \mathrm{ps}$, and a slower one around 0.7-1 ns. Due to our limited time window, we are not able to characterize the 2 to $3 \mathrm{~ns}$ component expected for the global tumbling of the DNA sequences. We find that the orientational dynamics of 2-AP within the hairpin loop are not site dependent.
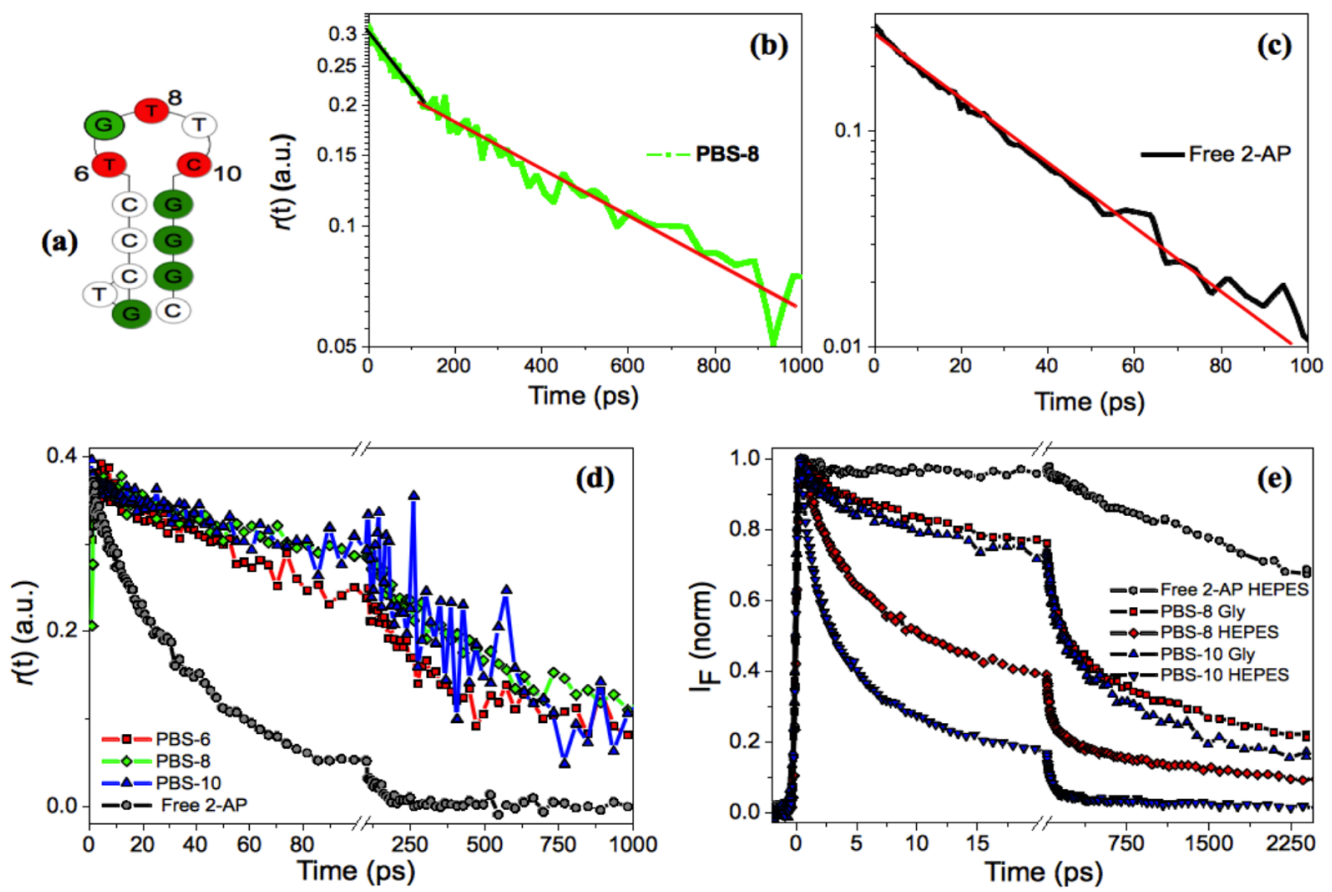

Fig. 1. (a) Schematic structure of $\triangle \mathrm{P}(-) \mathrm{PBS}$ : one of the three nucleobases in position 6,8 or 10 is replaced by 2-AP. (b) and (c) Anisotropy decay, in logarithmic scale of $\triangle \mathrm{P}(-) \mathrm{PBS}$ labelled at position 8 , and of free 2-AP respectively. (d) Comparison of the anisotropy decays of free 2-AP (grey), $\triangle \mathrm{P}(-) \mathrm{PBS}$ labelled at position 6 (red), 8 (green), and 10 (blue). (e) Comparison of normalized fluorescence decay of free 2-AP (grey), and of $\triangle \mathrm{P}(-) \mathrm{PBS}$ labelled at position, 8 (green) and 10 (blue) in aqueous solution, and in $70 \%$ glycerol solution. 
The slower $\approx 0.8$-ns anisotropy decay, contributes up to $55 \%$ to $70 \%$ of the total anisotropy amplitude. Given this time scale, it is attributed to motions of the hairpin loop. The faster 0.1-ns component is most probably due to locally restricted motion of 2-AP and represents the limiting time scale between static and dynamic fluorescence quenching, as was pointed out in recent studies $[1,3,5]$.

In order to further differentiate static from dynamic quenching, it is common to enhance the solvent viscosity, with the expectation that dynamic quenching is suppressed. Fig. 1e compares the magic angle fluorescence kinetic traces of two 2-AP-labelled nucleotides, in HEPES and in a (70/30) glycerol/water mixture. We observe that the fastest, static quenching components are strongly reduced in amplitude, indicating that enhanced hydrogen-bonding interactions with the solvent significantly perturb stacking interactions of 2-AP with its nearest neighbours, as suggested for similar glycerol-induced destabilisation effects observed for single stranded DNA [7]. In addition, the residual quenching dynamics is no longer site-specific in the presence of glycerol, indicating that the interactions with the solvent dominate.

\section{Conclusions}

We have shown that 2-aminopurine is a convenient fluorescence probe for monitoring the dynamics of a DNA hairpin. In particular down-conversion fluorescence anisotropy is a powerful tool for disentangling the multiple exponential components found in the isotropic fluorescence decay of 2-AP-labeled oligonucleotides. We have identified two time scales for the orientational dynamics of 2-AP. A 0.1 -ns one most probably related to depolarizing motion restricted by interactions with neighbouring bases, and a slower $\approx 0.8 \mathrm{~ns}$ one assigned to dynamics of the hairpin loop. No anisotropy decay faster than $100 \mathrm{ps}$ is observed, meaning that the sub-100ps quenching processes are due to static structures (e.g. stacking interactions). Besides, we have confirmed that the dominant effect of glycerol is to destabilize the local interaction of 2-AP with its nearest neighbours. This is evidenced by a strong reduction of the fastest static quenching components preferentially probed with the present high time resolution. It complements nanosecond experiments highlighting a slowdown of the collisional quenching processes related to the higher viscosity of glycerol [8].

The present study sets the basis for further femtosecond spectroscopy and molecular dynamics studies to be conducted on $\triangle \mathrm{P}(-) \mathrm{PBS}$ bound to NCp7, a nucleocapsid protein of HIV-1 known to promote the crucial second strand transfer during reverse transcription.

\section{References}

1. H. Nguyen, L. Zhao, C.W. Gray, D.M. Gray, and T. Xia, Biochemistry 50 (42), 8989, (2011)

2. C. Wan, T. Xia, H. Becker, A.H. Zewail, Chem. Phys. Let., 412, 1-3, 158 , (2005)

3. O.J.G. Somsen, G. Trinkunas, M.N. de Keijzer, A. van Hoek, H. van Amerongen, J. Lumin., 119-120, 100, (2006)

4. J. Léonard, T. Gelot, K. Torgasin and S. Haacke, J. Phys.: Conf. Ser. 277 012017, (2011)

5. J. Godet, N. Ramalanjaona, K.K. Sharma, L. Richert, H. de Rocquigny, J-L. Darlix, G. Duportail, and Y. Mély, Nucl. Acids Res., 39, 6633, (2011)

6. T. Gelot, P. Tourón-Touceda, O. Crégut, J. Léonard and S. Haacke, J. Phys. Chem. A 116, 2819, (2012)

7. G. Bonner, A.M. Klibanov, Biotechnol. Bioeng., 68 (3), 339, (2000)

8. S.V. Avilov, E. Piemont, V. Shvadchak, H. De Rocquigny, Y. Mély, Nucl. Acids Res., 36, 885, (2008) 